\title{
Bipartite Consensus of Heterogeneous Multiagent Systems with Diverse Input Delays
}

\author{
Hongtao Ye $\mathbb{C D}^{1,2,3}$ Zhongqiu Chen, ${ }^{1}$ Wenguang Luo, ${ }^{3}$ Jiayan Wen, ${ }^{3}$ and Kene $\mathrm{Li}^{1}$ \\ ${ }^{1}$ School of Electrical and Information Engineering, Guangxi University of Science and Technology, Liuzhou 545036, China \\ ${ }^{2}$ Guangxi Key Laboratory of Automatic Detecting Technology and Instruments, Guilin University of Electronic Technology, \\ Guilin 541004, China \\ ${ }^{3}$ Guangxi Key Laboratory of Automobile Components and Vehicle Technology, Guangxi University of Science and Technology, \\ Liuzhou 545036, China
}

Correspondence should be addressed to Hongtao Ye; yehongtao@126.com

Received 7 May 2020; Accepted 29 June 2020; Published 15 July 2020

Guest Editor: Shao-Bo He

Copyright (c) 2020 Hongtao Ye et al. This is an open access article distributed under the Creative Commons Attribution License, which permits unrestricted use, distribution, and reproduction in any medium, provided the original work is properly cited.

This paper investigates the bipartite consensus problem of heterogeneous multiagent systems with diverse input delays. Based on the systems composed of first-order and second-order agents, the novel control protocols are designed. Using frequency-domain analysis and matrix theory, the corresponding upper bounds of the allowable delays are obtained under the undirected topology and directed topology, respectively. Finally, simulation examples are given to verify the theoretical analysis.

\section{Introduction}

Multiagent systems and its cooperative control [1] are widely used in many practical systems such as unmanned aerial vehicles (UAV) [2], monitoring and security [3], sensor network [4], and delay system [5-11].

It is noteworthy that most of the problems about the consensus of multiagent systems are focused on cooperative network. In fact, cooperative and competitive relationships exist extensively in both natural and engineered network systems, such as opinion dynamics in social networks [12] and biological systems [13]. The bipartite consensus was firstly proposed in [14], which defined a signed graph the edges with positive and negative weights to describe the cooperative and competitive relations between agents. Bipartite consensus can be used for formation control [15], obstacle avoidance of wheeled robots [16], and nanoquadcopters formation [17].

There are many factors that affect the stability of agents. The time delay problem is one of the important problems that affect the consensus of a multiagent system. The bipartite consensus problem of second-order multiagent systems with fixed time delays was studied in [18]. Based on the second-order multiagent systems, Tian et al. [19] discussed the bipartite consensus problem of the system under different disturbances. The bipartite consensus with arbitrary finite communication delay was discussed in [20]. Most of the above work use undirected graphs as communication networks. Compared with undirected graphs [21], directed graphs [22-25] are more versatile and cost effective when the edge weights can be arbitrary between two agents.

This paper pays attention to bipartite consensus of heterogeneous MAS [26-28] with diverse input delays. As far as we know, there are few studies on this aspect, which is the motivation of this work. The theoretical analysis and simulation are presented under undirected topology and directed topology. The upper bounds of the allowable delays are given.

The rest of this article is structured as follows. Section 2 introduces some concepts and basic lemmas of graph theory. In Section 3, the bipartite consensus analysis of heterogeneous multiagent systems with multiple input delays under undirected and directed topologies is presented. In Section 4, two numerical examples are given to illustrate the validity of theoretical analysis. Finally, Section 5 draws some conclusions. 


\section{Preliminaries and Problem Formulation}

2.1. Preliminaries. In this section, the concepts and lemmas of some preliminary diagrams are introduced. We consider a heterogeneous multiagent system with $n$ agents. The connection between agents is represented by an undirected graph or directed graph $G=(V, \varepsilon, A)$, where $V=\left\{\zeta_{1}, \zeta_{2}, \ldots, \zeta_{n}\right\}$ represents the set of nodes, $\varepsilon \subseteq V \times V$ represents the set of edges, and $A=\left[a_{i j}\right] \in R^{n \times n}$ represents the adjacency matrix of $G$. As the multiagent system of cooperation and competition is studied in this paper, the value can be either positive or negative. Here, we choose $a_{i i} \neq 0$ for all $i \in\{1,2, \ldots, n\}$. If $a_{i j} \neq 0$, nodes $\zeta_{j}$ and $\zeta_{i}$ have information exchange; then, node $\zeta_{j}$ is said to be the neighbor of node $\zeta_{i}$. The set of neighbors of node $\zeta_{i}$ is denoted by $N_{i}=\left\{\zeta_{j} \mid\left(\zeta_{j}, \zeta_{i}\right) \in \varepsilon\right\}$. Graph $G$ is strongly connected if there is a path between any two nodes in graph $G$. The Laplacian matrix of $G$ is defined as $L_{s}=C-A$, where $C=\operatorname{diag}\left\{\sum_{j=1}^{n}\left|a_{1 j}\right|, \sum_{j=1}^{n}\left|a_{2 j}\right|, \ldots, \sum_{j=1}^{n}\left|a_{n j}\right|\right\}$. Therefore, the elements of Laplacian matrix $L_{s}$ are

$$
L_{s}= \begin{cases}\sum_{\zeta_{i} \in N_{i}}\left|a_{i j}\right|, & j=i, \\ -a_{i j}, & j \neq i .\end{cases}
$$

Assumption 1 (see [29]). $\sum_{j \in N_{l}}\left|a_{i j}\right|=0, \forall i \in N_{i}$, and $\sum_{j \in N_{i}}\left|a_{i j}\right|=0, \forall i \in N_{l}$.

Lemma 1 (see [30]). For Assumption 1, Laplace's matrix $L_{s}$ has at least two roots of zero. Give the time-delay system

$$
\dot{y}(t)=\sum_{i=1}^{N} A_{i} y\left(t-\tau_{i}\right),
$$

where $y(t) \in R^{n}, A_{i} \in R^{n \times n}, \tau_{i} \in R$, and $N$ is a positive integer.

Taking the Laplace transform, we can get the characteristic equation:

$$
\operatorname{det}\left(s I-\sum_{i=1}^{N} A_{i} y\left(t-\tau_{i}\right)\right)=0 .
$$

Lemma 2 (see [30]). According to the characteristic equation of Lemma 1, if it has only two zero roots and the rest of its roots are on the left half-plane of the complex plane, then it has $\lim _{t \rightarrow \infty} y(t)=\alpha+\beta t$, where $\alpha \in R^{n}$ and $\beta \in R^{n}$ are constant vectors.

Lemma 3 (see [31]). Let $h(\lambda)=\arctan (\delta \mu(\lambda) / \mu(\lambda))$, where $\delta>0$. When $\lambda>0, \mu(\lambda)>0$ increases monotonically; then, $h(\lambda)$ decreases monotonically.

Lemma 4 (see [31]). $y /\left(1+y^{2}\right)<\arctan (y)$ holds for $y>0$.

Lemma 5 (see [30]). If $m>0$, then the inequality $y /\left(1+y^{2}\right)<\arctan (y)+m$ holds for $y>0$. If $-(\pi / 2)<m<0$, then there exists $y_{0}>0$, such that the inequality $y /\left(1+y^{2}\right)<\arctan (y)+m$ holds for $y>y_{0}$.
Lemma 6 (see [32]). If and only if the connected graph $G$ has a globally accessible node, Laplace's matrix $L_{s}$ has a simple root 0 . The corresponding right eigenvector is $1_{n}=[1, \ldots, 1]^{T}$, that is, $L 1_{\mathbf{n}}=0$.

2.2. Problem Formulation. In this section, we will consider a heterogeneous multiagent system composed of agents, where $m$ agents are second-order agents and $n-m$ agents are first-order agents. The information transmission of each agent in the heterogeneous system is represented by $G$, and each agent represents a node.

Suppose the input delay of each agent in the system is not consistent. The dynamics of each second-order agent is given as follows:

$$
\left\{\begin{array}{l}
\dot{x}_{i}(t)=v_{i}(t), \\
\dot{v}_{i}(t)=u_{i}\left(t-T_{i}\right) \quad i=1,2, \ldots, m,
\end{array}\right.
$$

where $x_{i}, v_{i}, u_{i} \in R$ represent the position, velocity, and control input of the second-order agent $i$, respectively. $T_{i}>0$ represents the input delay. The dynamics of each first-order agent is given as follows:

$$
\dot{x}_{l}=u_{l}\left(t-T_{l}\right) \quad l=m+1, m+2, \ldots, n,
$$

where $x_{l}, u_{l} \in R$ represent the position and control input of the first-order agent $l$, respectively. $T_{l}>0$ represents the input delay.

The bipartite consensus means all agents converge to a value which is the same for all in modulus but not in sign through distributed protocols. Similar to [33], the bipartite consensus protocols for the second-order agents are given by

$$
\begin{aligned}
u_{i}= & k_{1} \sum_{j \in N_{i} \cup N_{l}}\left|a_{i j}\right|\left(\operatorname{sgn}\left(a_{i j}\right) x_{j}-x_{i}\right) \\
& +k_{2} \sum_{j \in N_{i} \cup N_{l}}\left|a_{i j}\right|\left(\operatorname{sgn}\left(a_{i j}\right) v_{j}-v_{i}\right) \quad i=1,2, \ldots, m,
\end{aligned}
$$

where $k_{1}, k_{2}>0$.

Based on the dynamic neighbor estimation rule in [31, 34-36], an estimated speed is added to the first-order agent. The bipartite consensus protocols are given by

$$
\begin{aligned}
& \dot{u}_{l}=v_{l}+k_{2} \sum_{j \in N_{i} \cup N_{l}}\left|a_{l j}\right|\left(\operatorname{sgn}\left(a_{l j}\right) x_{j}-x_{l}\right) \\
& \dot{v}_{l}=k_{1} \sum_{j \in N_{i} \cup N_{l}}\left|a_{l j}\right|\left(\operatorname{sgn}\left(a_{l j}\right) x_{j}-x_{l}\right) \quad l=m+1, m+2, \ldots, n,
\end{aligned}
$$

where $k_{1}, k_{2}>0$.

The main purpose of this paper is to study the bipartite consensus protocol for heterogeneous multiagents with diverse input delays under the undirected topology and directed topology, respectively.

\section{Main Results}

3.1. Bipartite Consensus under Undirected Topology. We will consider the bipartite consensus of heterogeneous multiagent systems with diverse input delays under undirected 
topology in this section. This paper uses neighborhood estimation rules to estimate the speed of first-order agents. Using the dynamic change of the position of the agent around the first-order agents, the estimated velocity is substituted for its actual velocity. Based on the heterogeneous system with both cooperative and competitive relations, the second-order agent control protocol is designed as follows:

$$
\begin{aligned}
\dot{x}_{i}(t)= & v_{i}(t), \\
\dot{v}_{i}(t)= & k_{1} \sum_{j \in N_{i} \cup N_{l}}\left|a_{i j}\right|\left(\operatorname{sgn}\left(a_{i j}\right) x_{j}\left(t-\tau_{i}\right)-x_{i}\left(t-\tau_{i}\right)\right) \\
& +k_{2} \sum_{j \in N_{i} \cup N_{l}}\left|a_{i j}\right|\left(\operatorname{sgn}\left(a_{i j}\right) v_{j}\left(t-\tau_{i}\right)-v_{i}\left(t-\tau_{i}\right)\right), \\
i & =1,2, \ldots, m,
\end{aligned}
$$

and the first-order agent control protocol is designed as follows:

$$
\begin{gathered}
\dot{x}_{l}(t)=v_{l}\left(t-\tau_{l}\right)+k_{2} \sum_{j \in N_{i} \cup N_{l}}\left|a_{l j}\right|\left(\operatorname{sgn}\left(a_{l j}\right) x_{j}\left(t-\tau_{l}\right)-x_{l}\left(t-\tau_{l}\right)\right), \\
\dot{v}_{l}(t)=k_{1} \sum_{j \in N_{i} \cup N_{l}}\left|a_{l j}\right|\left(\operatorname{sgn}\left(a_{l j}\right) x_{j}(t)-x_{l}(t)\right), \\
l=m+1, m+2, \ldots, n,
\end{gathered}
$$

where $\operatorname{sgn}(\cdot)$ is the sign function, $k_{1}>0$ and $k_{2}>0$ are the control gains, $N_{i}$ and $N_{l}$ represent the neighborhood of the agent $i$ and $l$, and $\tau_{i}$ and $\tau_{l}$ represent the input delay of the agent $i$ and $l$.

Lemma 7. For the heterogeneous multiagent systems (8) and (9), if and only if the Laplacian matrix $L_{s}$ has at least one zero eigenvalue, the real parts of the rest of eigenvalues are positive, and satisfy the condition of inequality (10), and the systems can achieve bipartite consensus.

$$
\frac{\lambda_{i} \sqrt{k_{1}^{2}+\omega_{c i}^{2} k_{2}^{2}}}{\omega_{c i}^{2}}<1,
$$

where $\lambda_{i}$ is the eigenvalue of $L_{s}$ and $\omega_{c i}$ satisfies $\omega_{c i}=$ $\arctan \left(\omega_{c i} k_{2} / k_{1}\right)$.

Proof. Take the Laplace transform on (8) and (9); then, we can obtain

$$
\begin{aligned}
s X_{i}(s)= & V_{i}(s), \\
s V_{i}(s)= & k_{1} \sum_{j \in N_{i} \cup N_{l}}\left|a_{i j}\right|\left(\operatorname{sgn}\left(a_{i j}\right) X_{j}(s)-X_{i}(s)\right) e^{-\tau_{i} s} \\
& +k_{2} \sum_{j \in N_{i} \cup N_{l}}\left|a_{i j}\right|\left(\operatorname{sgn}\left(a_{i j}\right) V_{j}(s)-V_{i}(s)\right) e^{-\tau_{i} s}, \\
& i=1,2, \cdots, m,
\end{aligned}
$$

$$
\begin{gathered}
s X_{l}(s)=V_{l}(s) e^{-\tau_{l} s}+k_{2} \sum_{j \in N_{i} \cup N_{l}}\left|a_{l j}\right|\left(\operatorname{sgn}\left(a_{l j}\right) X_{j}(s)-X_{l}(s)\right) e^{-\tau_{l} s}, \\
s V_{l}(s)=k_{1} \sum_{j \in N_{i} \cup N_{l}}\left|a_{l j}\right|\left(\operatorname{sgn}\left(a_{l j}\right) X_{j}(s)-X_{l}(s)\right), \\
\quad l=m+1, m+2, \ldots, n .
\end{gathered}
$$

From (11) and (12), we can obtain

$$
s^{2} X(s)=-\left(k_{1}+k_{2} s\right) X(s) L_{s} e^{-\tau s},
$$

where $\mathbf{X}(s)=\left[X_{1}(s), X_{2}(s), \ldots, X_{n}(s)\right]^{T}$ is the Laplace transform of $\mathbf{x}(t)=\left[x_{1}(t), x_{2}(t), \ldots, x_{n}(t)\right]^{T}$. Thus, the characteristic equation of (8) and (9) is given by

$$
\operatorname{det}\left(s^{2} I+\left(k_{1}+k_{2} s\right) L_{s} e^{-\tau s}\right)=0 .
$$

Because the topology is undirected connected, 0 is a simple root of matrix $L_{s}$, and the rest of the roots are positive. Hence, $\operatorname{rank}\left(L_{s}\right)=n-1$. Let $\lambda_{i}, i=1,2, \ldots, n$ be the eigenvalue of $L_{s}$. Assume $\lambda_{1}=0, \lambda_{i}>0, i=2,3, \ldots, n$, then (14) is equal to

$$
s^{2} \prod_{i=2, \ldots, n}\left(s^{2}+\lambda_{i}\left(k_{1}+k_{2} s\right) e^{-\tau s}\right)=0 .
$$

So, the equation has two roots of zero. Analyze the rest of the roots of the equation. For

$$
s^{2}+\lambda_{i}\left(k_{1}+k_{2} s\right) e^{-\tau s}=0, \quad i=2,3, \ldots, n,
$$

let $f(s)=1+g_{i}(j \omega)=0$. Equation (16) can be written as $1+\lambda_{i}\left(k_{1}+k_{2} s\right) e^{-\tau s} / s^{2}=0$, where $g_{i}(j \omega)=\lambda_{i}\left(k_{1}+k_{2} s\right)$ $e^{-\tau s} / s^{2}$. Based on the Nyquist criterion, if and only if the curve $g_{i}(j \omega)$ does not include the point $(-1, j 0)$, the characteristic root of equation (16) is located on the left halfplane of the complex plane. Then,

$$
g_{i}(j \omega)=\frac{\lambda_{i} \sqrt{k_{1}^{2}+\omega^{2} k_{2}^{2}}}{\omega^{2}} e^{-j\left(\omega \tau+\pi-\arctan \left(\omega k_{2} / k_{1}\right)\right)} .
$$

Then,

$$
\left|g_{i}(j \omega)\right|=\frac{\left|\lambda_{i}\right| \sqrt{k_{1}^{2}+\omega_{c i}^{2} k_{2}^{2}}}{\omega_{c i}^{2}}
$$

$$
\arg \left(g_{i}(j \omega)\right)=-\omega \tau+\arctan \left(\omega k_{2} / k_{1}\right)
$$

where $\arg (\cdot)$ represents the phase. We know that when $\omega \in(0,+\infty),\left|g_{i}(j \omega)\right|$ is monotonically decreasing. When curve $g_{i}(j \omega)$ crosses the real axis for the first time, $\omega_{c i}$ satisfies $-\omega \tau+\arctan \left(\omega k_{2} / k_{1}\right)=0$. Therefore,

$$
\left|g_{i}(j \omega)\right|=\frac{\left|\lambda_{i}\right| \sqrt{k_{1}^{2}+\omega_{c i}^{2} k_{2}^{2}}}{\omega_{c i}^{2}}<1 \text {. }
$$

In other words, (10) is true. Therefore, except for the two zero roots, all the characteristic roots of (14) are located in the left half-plane. Based on Lemma 2, it is true that $\lim _{t \rightarrow \infty} x(t)=\alpha+\beta t$, where $\alpha=\left[\alpha_{1}, \alpha_{2}, \ldots, \alpha_{n}\right]^{T} \in R^{n}$ and $\beta=\left[\beta_{1}, \beta_{2}, \cdots, \beta_{n}\right]^{T} \in R^{n}$ are constant vectors. From the 
control protocols (8) and (9), it is true that $\lim _{t \rightarrow \infty} v(t)=\beta$, where $\mathbf{v}(t)=\left[v_{1}(t), v_{2}(t), \ldots, v_{n}(t)\right]^{T}$. For $(8)$,

$$
\begin{aligned}
k_{1} & \sum_{j \in N_{i} \cup N_{l}}\left|a_{i j}\right|\left(\alpha_{j}-\alpha_{i}\right)+\left(k_{1}(t-\tau)+k_{2}\right) \\
& \sum_{j \in N_{i} \cup N_{l}}\left|a_{i j}\right|\left(\beta_{j}-\beta_{i}\right)=0, \quad i=1,2, \ldots, m .
\end{aligned}
$$

And (20) is also true for any $t$, and we have

$$
\begin{gathered}
\sum_{j \in N_{i} \cup N_{l}}\left|a_{i j}\right|\left(\alpha_{j}-\alpha_{i}\right)=0, \quad i=1,2, \ldots, m . \\
\sum_{j \in N_{i} \cup N_{l}}\left|a_{i j}\right|\left(\beta_{j}-\beta_{i}\right)=0, \quad i=1,2, \ldots, m .
\end{gathered}
$$

For (9),

$$
\begin{array}{r}
\sum_{j \in N_{i} \cup N_{l}}\left|a_{l j}\right|\left(\alpha_{j}-\alpha_{l}\right)+t \sum_{j \in N_{i} \cup N_{l}}\left|a_{l j}\right|\left(\beta_{j}-\beta_{l}\right)=0, \\
l=m+1, m+2, \ldots, n .
\end{array}
$$

And (23) is also true for any $t$, and we have

$$
\begin{gathered}
\sum_{j \in N_{i} \cup N_{l}}\left|a_{l j}\right|\left(\alpha_{j}-\alpha_{l}\right)=0, \quad l=m+1, m+2, \ldots, n, \\
\sum_{j \in N_{i} \cup N_{l}}\left|a_{l j}\right|\left(\beta_{j}-\beta_{l}\right)=0, \quad l=m+1, m+2, \ldots, n .
\end{gathered}
$$

From (21) and (24), it is true that $L \alpha=0$. From (22) and (25), it is true that $L \beta=0$. Because $\operatorname{rank}(L)=n-1$ and $L[1, \ldots, 1]^{T}=0$. According to Lemma 6 , we have $\alpha=a[1, \ldots, 1]^{T}, \beta=b[1, \ldots, 1]^{T}$.

Theorem 1. For the heterogeneous multiagent systems (8) and (9) under undirected communication topology, the systems can achieve bipartite consensus if the largest input delay satisfies $\tau_{\max }<\tau^{*}$, where $\tau^{*}=\arctan$ $\left(k_{2} / k_{1} \sqrt{\left(\lambda_{\max }^{2} k_{2}^{2}+\sqrt{\lambda_{\max }^{4} k_{2}^{4}+4 \lambda_{\max }^{2} k_{1}^{2}} / 2\right)}\right) /$ $\sqrt{\left(\lambda_{\max }^{2} k_{2}^{2}+\sqrt{\lambda_{\max }^{4} k_{2}^{4}+4 \lambda_{\max }^{2} k_{1}^{2}} / 2\right)}$, and $\lambda_{\max }$ is the maximum eigenvalue of $L_{s}$.

Proof. For Lemma 7, we can get $\omega_{c i} \tau=\arctan \left(\omega_{c i} k_{2} / k_{1}\right)$. Namely,

$$
\tau=\frac{\arctan \left(\omega_{c i} k_{2} / k_{1}\right)}{\omega_{c i}} .
$$

Taking the derivative of $\tau$ with respect to $\omega_{c i}$ for equation (24), we can obtain

$$
\frac{d \tau}{d \omega_{c i}}=\frac{1}{\omega_{c i}^{2}}\left(\frac{\eta \omega_{c i}}{1+\left(\eta \omega_{c i}\right)^{2}}-\arctan \left(\eta \omega_{c i}\right)\right),
$$

where $\eta=k_{2} / k_{1}$. According to Lemma $4, \tau$ decreases as $\omega_{c i}$ increases.

Then,

$$
\omega_{c i}>\sqrt{\frac{\lambda_{i}^{2} k_{2}^{2}+\sqrt{\lambda_{i}^{4} k_{2}^{4}+\lambda_{i}^{2} k_{1}^{2}}}{2}} .
$$

From (26), (28), and Lemma 3, we can obtain

$$
\tau^{*}<\frac{\arctan \left(\left(k_{2} / k_{1}\right) \sqrt{\left(\lambda_{\max }^{2} k_{2}^{2}+\sqrt{\lambda_{\max }^{4} k_{2}^{4}+4 \lambda_{\max }^{2} k_{1}^{2}}\right) / 2}\right)}{\sqrt{\left(\lambda_{\max }^{2} k_{2}^{2}+\sqrt{\lambda_{\max }^{4} k_{2}^{4}+4 \lambda_{\max }^{2} k_{1}^{2}}\right) / 2}},
$$

where $\lambda_{\max }$ is the maximum eigenvalue of $L_{s}$.

3.2. Bipartite Consensus under Directed Topology. We will study the bipartite consensus of heterogeneous multiagent systems with diverse input delays under directed topology in this section. The control protocols are designed as follows:

$$
\begin{aligned}
\dot{x}_{i}(t)= & v_{i}(t), \\
\dot{v}_{i}(t)= & k_{1} \sum_{j \in N_{i}}\left|a_{i j}\right|\left(\operatorname{sgn}\left(a_{i j}\right) x_{j}\left(t-\tau_{i}\right)-x_{i}\left(t-\tau_{i}\right)\right)+k_{1} \sum_{j \in N_{l}}\left|a_{i j}\right| \operatorname{sgn}\left(a_{i j}\right) x_{j}\left(t-\tau_{i}\right) \\
& +k_{2} \sum_{j \in N_{i}}\left|a_{i j}\right|\left(\operatorname{sgn}\left(a_{i j}\right) v_{j}\left(t-\tau_{i}\right)-v_{i}\left(t-\tau_{i}\right)\right)+k_{2} \sum_{j \in N_{l}}\left|a_{i j}\right| \operatorname{sgn}\left(a_{i j}\right) v_{j}\left(t-\tau_{i}\right), \quad i=1,2, \ldots, m, \\
\dot{x}_{l}(t)= & v_{l}\left(t-\tau_{l}\right)+k_{2} \sum_{j \in N_{l}}\left|a_{l j}\right|\left(\operatorname{sgn}\left(a_{l j}\right) x_{j}\left(t-\tau_{l}\right)-x_{l}\left(t-\tau_{l}\right)\right) \\
& +k_{2} \sum_{j \in N_{i}}\left|a_{l j}\right| \operatorname{sgn}\left(a_{l j}\right) x_{j}\left(t-\tau_{l}\right), \\
\dot{v}_{l}(t)= & k_{1} \sum_{j \in N_{l}}\left|a_{l j}\right|\left(\operatorname{sgn}\left(a_{l j}\right) x_{j}(t)-x_{l}(t)\right)+k_{1} \sum_{j \in N_{i}}\left|a_{l j}\right| \operatorname{sgn}\left(a_{l j}\right) x_{j}(t), \quad l=m+1, m+2, \ldots, n,
\end{aligned}
$$

where $k_{1}>0, k_{2}>0$ are the control gains, $N_{i}$ and $N_{l}$ represent the neighborhood of the agent $i$ and $l$, and $\tau_{i}$ and $\tau_{l}$ represent the input delay of the agent $i$ and $l$.
Lemma 8. For the heterogeneous multiagent systems (30) and (31), if and only if the Laplacian matrix $L_{s}$ has at least two zero eigenvalues, the real parts of the rest of eigenvalues 
are positive, and satisfy the condition of inequality (32), and the systems can achieve bipartite consensus:

$$
\frac{\lambda_{i} \sqrt{k_{1}^{2}+\omega_{c i}^{2} k_{2}^{2}}}{\omega_{c i}^{2}}<1,
$$

where $\lambda_{i}$ is the eigenvalue of $L_{s}$, and $\omega_{c i}$ satisfies

$$
\omega_{c i} \tau=\arctan \left(\frac{\omega_{c i} k_{2}}{k_{1}}\right)+\arctan \left(\frac{\operatorname{Im}\left(\lambda_{i}\right)}{\operatorname{Re}\left(\lambda_{i}\right)}\right) .
$$

Proof. The Laplace transform of (30) and (31):

$$
\begin{aligned}
s X_{i}(s)= & V_{i}(s), \\
s V_{i}(s)= & k_{1} \sum_{j \in N_{i}}\left|a_{i j}\right|\left(\operatorname{sgn}\left(a_{i j}\right) X_{j}(s)-X_{i}(s)\right) e^{-\tau_{i} s}+k_{1} \sum_{j \in N_{l}}\left|a_{i j}\right|\left(\operatorname{sgn}\left(a_{i j}\right) X_{j}(s)\right) e^{-\tau_{i} s} \\
& +k_{2} \sum_{j \in N_{i}}\left|a_{i j}\right|\left(\operatorname{sgn}\left(a_{i j}\right) V_{j}(s)-V_{i}(s)\right) e^{-\tau_{i} s}+k_{2} \sum_{j \in N_{l}}\left|a_{i j}\right|\left(\operatorname{sgn}\left(a_{i j}\right) X_{j}(s)\right) e^{-\tau_{i} s}, \quad i=1,2, \ldots, m, \\
s X_{l}(s)= & V(s) e^{-\tau_{l} s}+k_{2} \sum_{j \in N_{l}}\left|a_{l j}\right|\left(\operatorname{sgn}\left(a_{l j}\right) X_{j}(s)-X_{l}(s)\right) e^{-\tau_{l} s} \\
& +k_{2} \sum_{j \in N_{i}}\left|a_{l j}\right| \operatorname{sgn}\left(a_{l j}\right) X_{j}(s) e^{-\tau_{l} s}, \\
s V_{l}(s)= & k_{1} \sum_{j \in N_{l}}\left|a_{l j}\right|\left(\operatorname{sgn}\left(a_{l j}\right) X_{j}(s)-X_{l}(s)\right)+k_{1} \sum_{j \in N_{i}}\left|a_{l j}\right| \operatorname{sgn}\left(a_{l j}\right) V_{j}(s), \quad l=m+1, m+2, \ldots, n .
\end{aligned}
$$

From Assumption 1, (34), and (35),

$$
s^{2} X(s)=-\left(k_{1}+k_{2} s\right) X(s) L_{s} e^{-\tau s} .
$$

Then, the characteristic root of systems (31) and (32) is

$$
\operatorname{det}\left(s^{2} I+\left(k_{1}+k_{2} s\right) L_{s} e^{-\tau s}\right)=0 .
$$

Let $\lambda_{i}, i=1,2, \ldots, n$ be the eigenvalue of $L_{s}$. Assume $\lambda_{1}=$ $\lambda_{2}=0, \lambda_{i}>0, i=3,4, \ldots, n$; then, (37) is equal to

$$
s^{4} \prod_{i=3, \ldots, n}\left(s^{2}+\lambda_{i}\left(k_{1}+k_{2} s\right) e^{-\tau s}\right)=0 .
$$

Obviously, we know that the equation has four zero roots, and then we can analyze the rest of the characteristic roots of the equation. For

$$
s^{2}+\lambda_{i}\left(k_{1}+k_{2} s\right) e^{-\tau s}=0, i=3,4, \ldots, n,
$$

let $h(s)=1+g_{i}(j \omega)=0$. Therefore, equation (39) can be written as $1+\lambda_{i}\left(k_{1}+k_{2} s\right) e^{-\tau s} / s^{2}=0$, where $g_{i}(j \omega)=\lambda_{i}$ $\left(k_{1}+k_{2} s\right) e^{-\tau s} / s^{2}$. Based on Nyquist's criterion, if and only if curve $g_{i}(j \omega)$ does not include point $(-1, j 0)$, the characteristic root of equation (39) is located on the left half-plane of the complex plane. Then,

$$
\begin{aligned}
g_{i}(j \omega)= & \frac{\left|\lambda_{i}\right| \sqrt{k_{1}^{2}+\omega_{c i}^{2} k_{2}^{2}}}{\omega_{c i}^{2}} \\
& \cdot e^{-j\left(\omega \tau+\pi-\arctan \left(\omega k_{2} / k_{1}\right)-\arctan \left(\operatorname{Im}\left(\lambda_{i}\right) / \operatorname{Re}\left(\lambda_{i}\right)\right)\right) .}
\end{aligned}
$$

Therefore,

$$
\begin{aligned}
\left|g_{i}(j \omega)\right| & =\frac{\left|\lambda_{i}\right| \sqrt{k_{1}^{2}+\omega_{c i}^{2} k_{2}^{2}}}{\omega_{c i}^{2}}, \\
\arg \left(g_{i}(j \omega)\right) & =-\omega \tau+\arctan \left(\frac{\omega k_{2}}{k_{1}}\right)+\arctan \left(\frac{\operatorname{Im}\left(\lambda_{i}\right)}{\operatorname{Re}\left(\lambda_{i}\right)}\right),
\end{aligned}
$$

where $\arg (\cdot)$ represents the phase. And it can be seen that $\left|g_{i}(j \omega)\right|$ monotonically decreases for $\omega \in(0,+\infty)$. When curve $\left|g_{i}(j \omega)\right|$ crosses the real axis for the first time, $\omega_{c i}$ satisfies

$-\omega \tau+\arctan \left(\omega k_{2} / k_{1}\right)+\arctan \left(\operatorname{Im}\left(\lambda_{i}\right) / \operatorname{Re}\left(\lambda_{i}\right)\right)=0$.

In addition, if the characteristic root of (37) falls on the left half-plane, there is

$$
\left|g_{i}(j \omega)\right|=\frac{\left|\lambda_{i}\right| \sqrt{k_{1}^{2}+\omega_{c i}^{2} k_{2}^{2}}}{\omega_{c i}^{2}}<1 .
$$

In other words,

$$
\frac{\left|\lambda_{i}\right| \sqrt{k_{1}^{2}+\omega_{c i}^{2} k_{2}^{2}}}{\omega_{c i}^{2}}<1 .
$$

Therefore, except for the four zero roots, all the characteristic roots of (37) are located in the left half-plane. According to Lemma 6 and Theorem 1, we can get $\alpha=$ $a[1, \ldots, 1]^{T}$ and $\beta=b[1, \ldots, 1]^{T}$.

Theorem 2. For the heterogeneous multiagent systems (30) and (31) under directed communication topology, the systems can achieve bipartite consensus if the largest input delay satisfies $\tau_{\max }<\tau^{*}=\min \left(\tau_{a}, \tau_{b}\right)$, where $\tau_{a}=\arctan \left(k_{2} / k_{1}\right.$ $\left.\sqrt{|\lambda|_{\max }^{2} k_{2}^{2}+\sqrt{|\lambda|_{\max }^{4} k_{2}^{4}+4|\lambda|_{\max }^{2} k_{1}^{2}} / 2}\right)+\theta_{\max } / \sqrt{|\lambda|_{\max }^{2} k_{2}^{2}+}$ $\sqrt{|\lambda|_{\max }^{4} k_{2}^{4}+4|\lambda|_{\max }^{2} k_{1}^{2}} / 2$.

$\theta_{\max }=\arctan \left(\operatorname{Im}\left(\lambda_{i}\right) / \operatorname{Re}\left(\lambda_{i}\right)\right),|\lambda|_{\max }$ is the maximum modulus of $\lambda_{i}, \tau_{b}=\arctan \left(\widetilde{\omega}_{\max } k_{2} / k_{1}\right)-\theta_{\max } / \widetilde{\omega}_{\max }, \widetilde{\omega}_{\max }=$ $\max _{3 \leq i \leq n}\left\{\widetilde{\omega}_{c i}\right\}, \quad \widetilde{\omega}_{c i}$ is the root of equation, $1 / \omega_{c i}\left(\eta \omega_{c i} / 1+\left(\eta \omega_{c i}\right)^{2}\right)-\quad \arctan \left(\eta \omega_{c i}\right)-\arctan \left(\operatorname{Im}\left(\lambda_{i}\right) /\right.$ $\left.\operatorname{Re}\left(\lambda_{i}\right)\right)=0$, and $\eta=k_{2} / k_{1}$. 


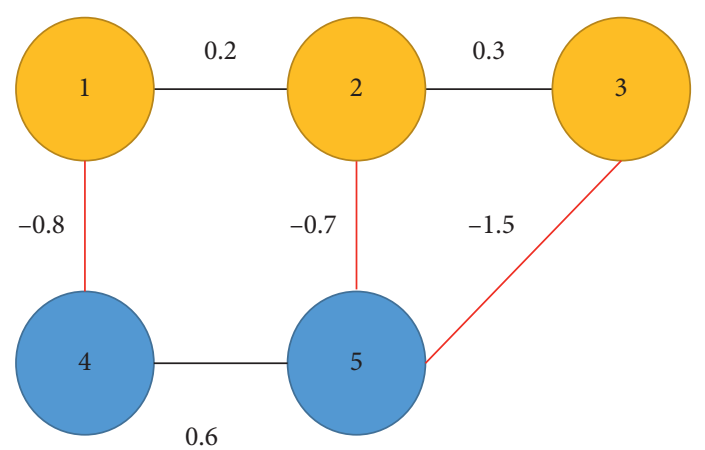

FIGURE 1: Undirected topology of a multiagent system.

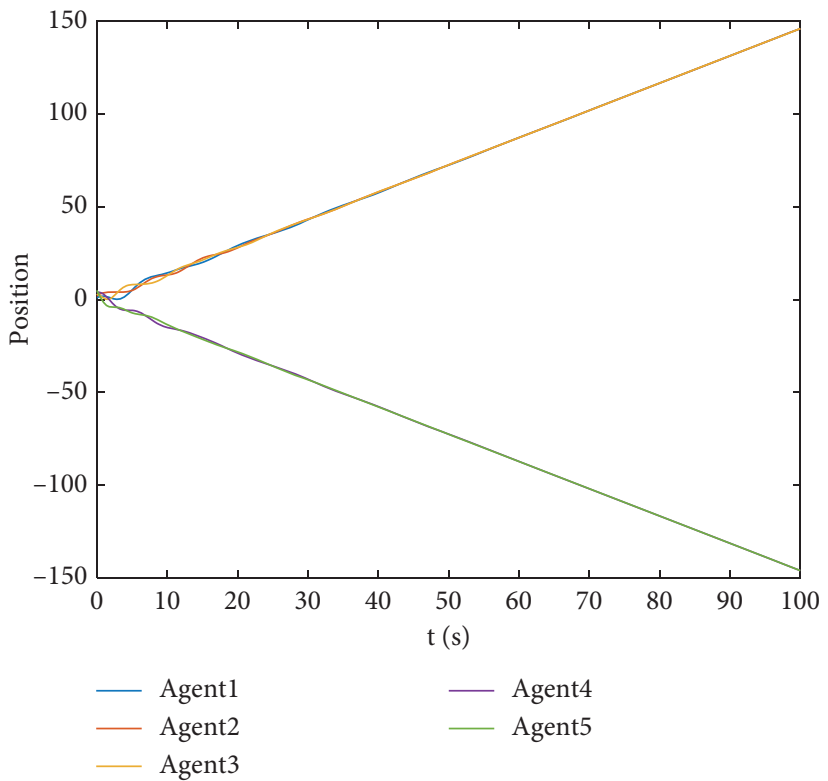

(a)

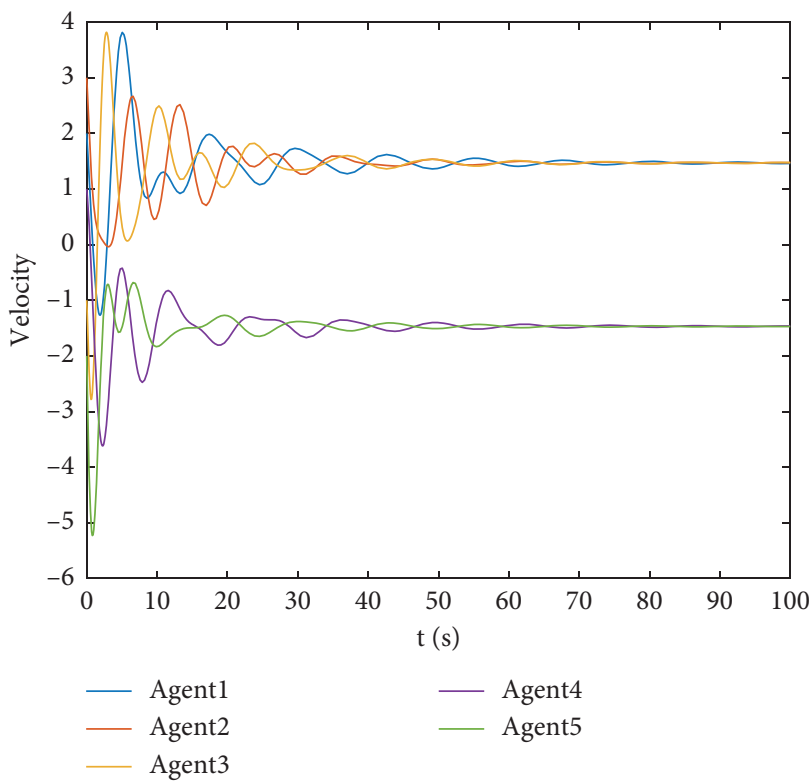

(b)

Figure 2: $\tau_{1}=0.5, \tau_{2}=0.4, \tau_{3}=0.3, \tau_{4}=0.2$, and $\tau_{5}=0.1$. (a) Position trajectories of all agents. (b) Velocity trajectories of all agents.

Proof. From (33), we can obtain

$$
\tau=\frac{\arctan \left(\omega_{c i} k_{2} / k_{1}\right)+\arctan \left(\operatorname{Im}\left(\lambda_{i}\right) / \operatorname{Re}\left(\lambda_{i}\right)\right)}{\omega_{c i}} .
$$

Taking the derivative of $\tau$ with respect to $\omega_{c i}$ for equation (44), we can obtain

$d \tau / d \omega_{c i}=\left(1 / \omega_{c i}^{2}\right)\left(\eta \omega_{c i} / 1+\left(\eta \omega_{c i}\right)^{2}-\arctan \left(\eta \omega_{c i}\right)-\right.$ $\left.\arctan \left(\operatorname{Im}\left(\lambda_{i}\right) / \operatorname{Re}\left(\lambda_{i}\right)\right)\right)$, where $\eta=k_{2} / k_{1}$.

According to Lemma 5, if $m=\arctan \left(\operatorname{Im}\left(\lambda_{i}\right) /\right.$ $\left.\operatorname{Re}\left(\lambda_{i}\right)\right)>0$, then $\omega_{c i}>0$ and $d \tau / d \omega_{c i}<0$. According to (32), we can obtain $\omega_{c i}>\sqrt{\left|\lambda_{i}\right|^{2} k_{2}^{2}+\sqrt{\left|\lambda_{i}\right|^{4} k_{2}^{4}+\left|\lambda_{i}\right|^{2} k_{1}^{2}} / 2}$.

Then, using Lemma 8 , we have. $\tau=\arctan \left(k_{2} / k_{1}\right.$ $\left.\sqrt{\left|\lambda_{i}\right|^{2} k_{2}^{2}+\sqrt{\left|\lambda_{i}\right|^{4} k_{2}^{4}+4\left|\lambda_{i}\right|^{2} k_{1}^{2}} / 2}\right)+\arctan \left(\operatorname{Im}\left(\lambda_{i}\right) / \operatorname{Re}\left(\lambda_{i}\right)\right) /$ $\sqrt{\left|\lambda_{i}\right|^{2} k_{2}^{2}+} \sqrt{\left|\lambda_{i}\right|^{4} k_{2}^{4}+4\left|\lambda_{i}\right|^{2} k_{1}^{2}} / 2$.

According to Lemma 5, if $-\pi / 2<m=$ $\arctan \left(\operatorname{Im}\left(\lambda_{i}\right) / \operatorname{Re}\left(\lambda_{i}\right)\right)<0$, then there exists $\widetilde{\omega}_{c i}>0$, when $\omega_{c i}>\widetilde{\omega}_{c i}$ and $d \tau / d \omega_{c i}<0 . \widetilde{\omega}_{c i}$ is the root of $\frac{1}{\omega_{c i}}\left(\eta \omega_{c i} / 1+\left(\eta \omega_{c i}\right)^{2}\right)-\arctan \left(\eta \omega_{c i}\right)-\arctan \left(\operatorname{Im}\left(\lambda_{i}\right) / \operatorname{Re}\left(\lambda_{i}\right)\right)=0$,

where $\eta=k_{2} / k_{1}$.

Similarly, using Lemma 8, we have $\tau=\arctan$ $\left(\omega_{c i} k_{2} / k_{1}\right)+\arctan \left(\operatorname{Im}\left(\lambda_{i}\right) / \operatorname{Re}\left(\lambda_{i}\right)\right) / \omega_{c i}$.

In conclusion, according to Lemma 3 , the maximum allowable upper bound of the system is $\tau^{*}=\min \left(\tau_{a}, \tau_{b}\right)$, where

$$
\begin{aligned}
& \tau_{a}= \frac{\arctan \left(\left(k_{2} / k_{1}\right) \sqrt{|\lambda|_{\max }^{2} k_{2}^{2}+\sqrt{|\lambda|_{\max }^{4} k_{2}^{4}+4|\lambda|_{\max }^{2} k_{1}^{2}} / 2}\right)+\theta_{\max }}{\sqrt{|\lambda|_{\max }^{2} k_{2}^{2}+\sqrt{|\lambda|_{\max }^{4} k_{2}^{4}+4|\lambda|_{\max }^{2} k_{1}^{2} / 2}}}, \\
& \tau_{b}=\frac{\arctan \left(\widetilde{\omega}_{\max } k_{2} / k_{1}\right)-\theta_{\max }}{\widetilde{\omega}_{\max }},
\end{aligned}
$$




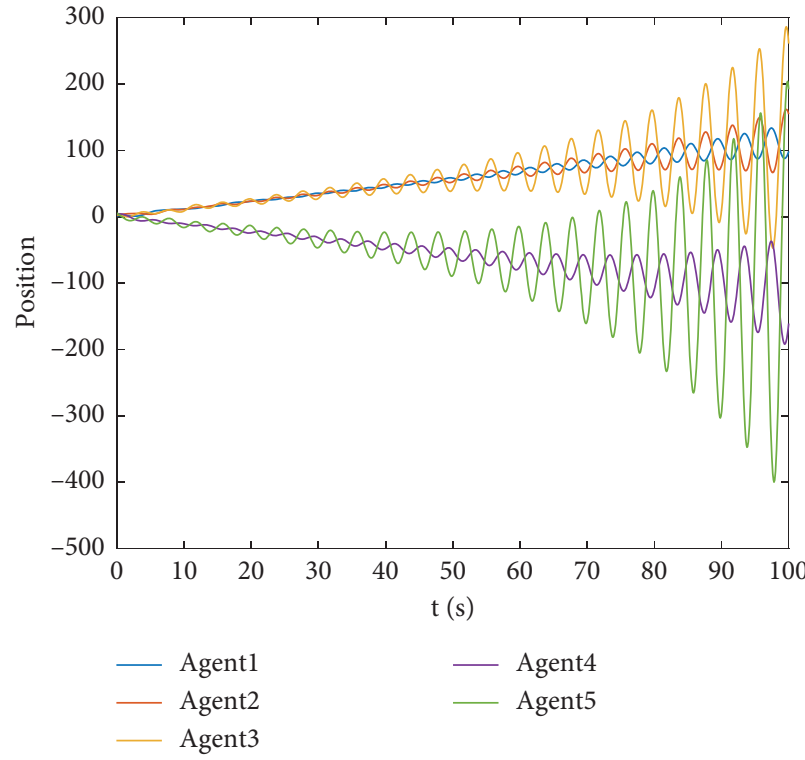

(a)

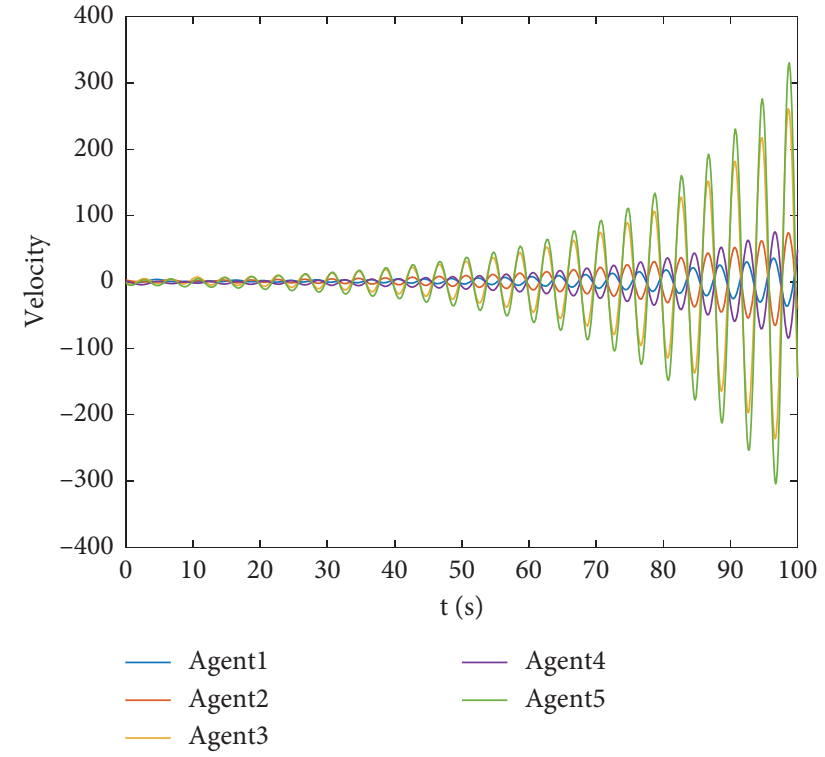

(b)

FIgURE 3: $\tau_{1}=0.4, \tau_{2}=0.3, \tau_{3}=0.4, \tau_{4}=0.3$, and $\tau_{5}=0.6$. (a) Position trajectories of all agents. (b) Velocity trajectories of all agents.

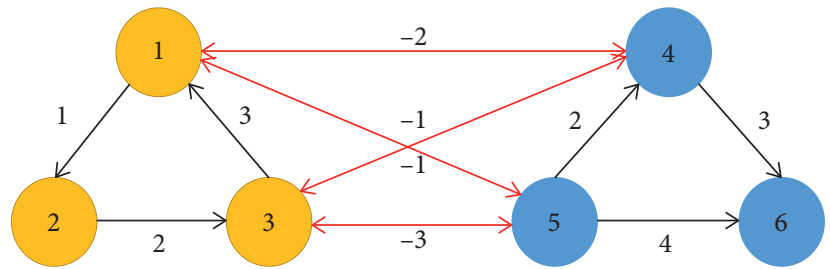

FIgURE 4: Directional topology of a multiagent system.

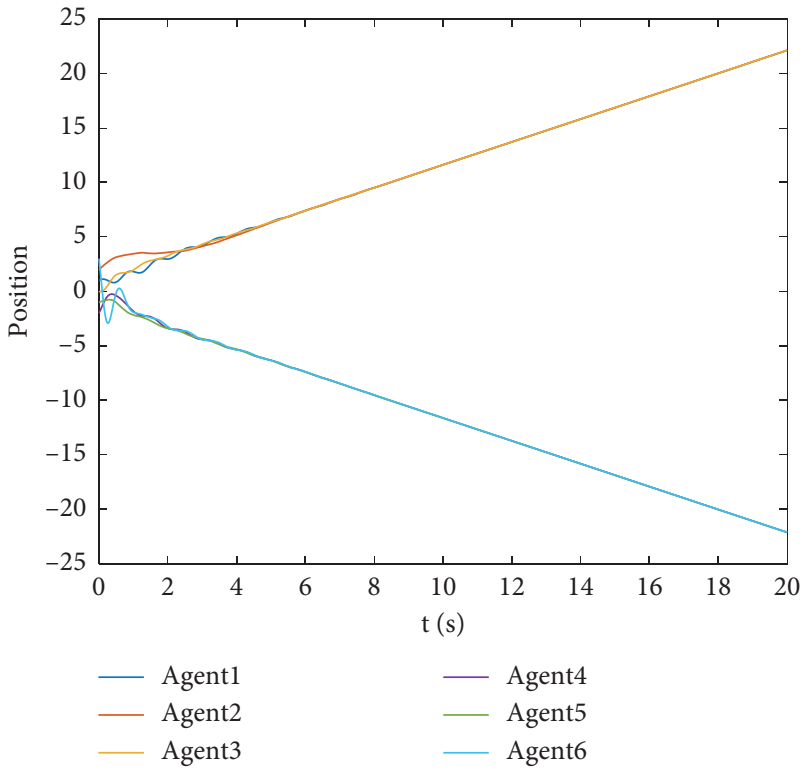

(a)

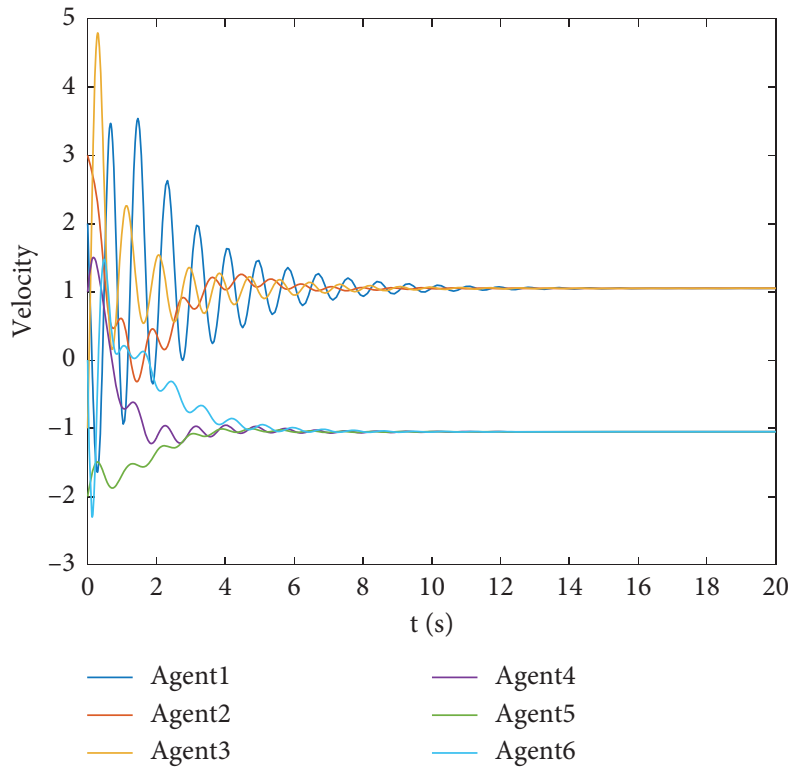

(b)

Figure 5: $\tau_{1}=0.17, \tau_{2}=0.16, \tau_{3}=0.15, \tau_{4}=0.14, \tau_{5}=0.13$, and $\tau_{6}=0.12$. (a) Position trajectories of all agents. (b) Velocity trajectories of all agents. 


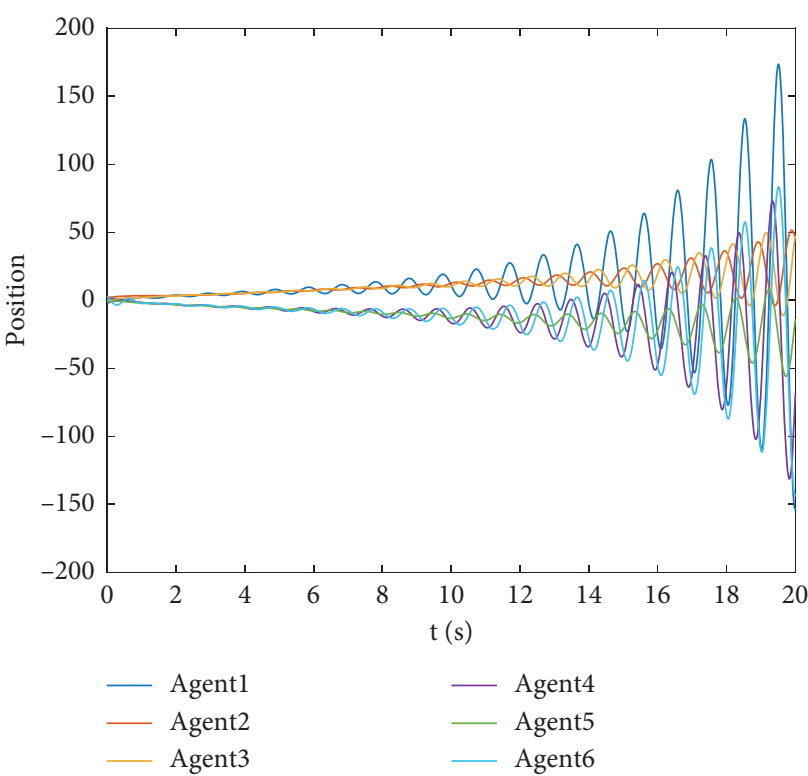

(a)

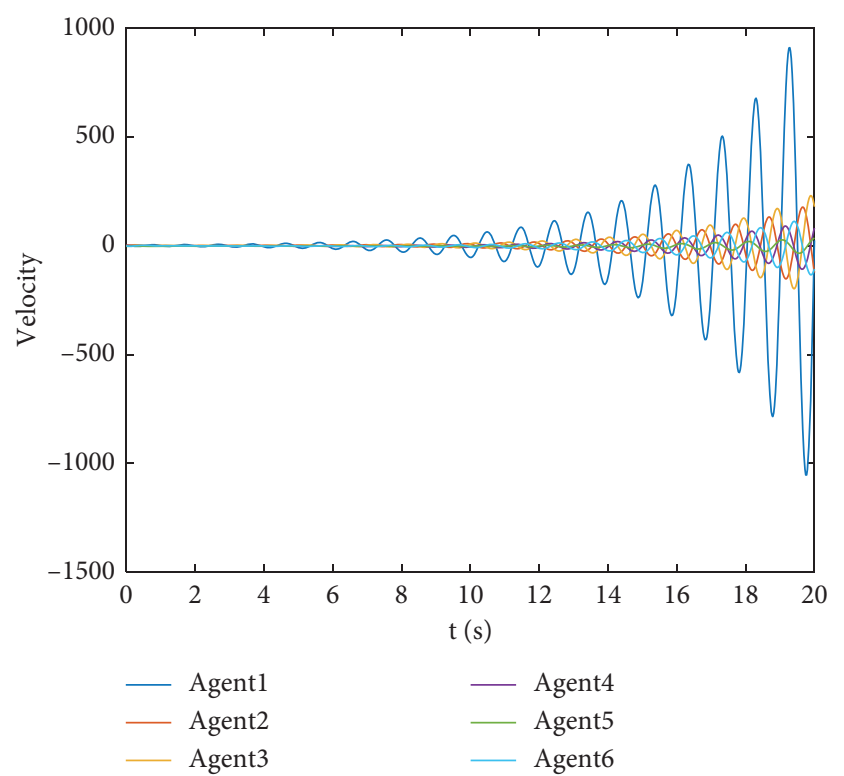

(b)

Figure 6: $\tau_{1}=0.22, \tau_{2}=0.14, \tau_{3}=0.15, \tau_{4}=0.16, \tau_{5}=0.15$, and $\tau_{6}=0.14$. (a) Position trajectories of all agents. (b) Velocity trajectories of all agents.

$$
\theta_{\max }=\arctan \left(\operatorname{Im}\left(\lambda_{i}\right) / \operatorname{Re}\left(\lambda_{i}\right)\right), \widetilde{\omega}_{\max }=\max _{3 \leq i \leq n}\left\{\widetilde{\omega}_{c i}\right\}, \widetilde{\omega}_{c i}
$$
is the root of equation $1 / \omega_{c i}\left(\eta \omega_{c i} / 1+\left(\eta \omega_{c i}\right)^{2}\right)-\arctan$ $\left(\eta \omega_{c i}\right)-\arctan \left(\operatorname{Im}\left(\lambda_{i}\right) / \operatorname{Re}\left(\lambda_{i}\right)\right)=0$, and $\eta=k_{2} / k_{1}$.

\section{Numerical Examples and Simulations}

Example 1. We will test and verify the results obtained by a multiagent system with five agents in this section. The initial conditions are randomly set and the topology is shown in Figure 1. The maximum eigenvalue of $L_{s}$ is $\lambda_{\max }=4.0445$. $k_{1}=0.45$ and $k_{2}=0.3$. According to Theorem 1 , we obtain $\tau^{*}<0.5057$. Let $\tau_{1}=0.5, \tau_{2}=0.4, \quad \tau_{3}=0.3, \tau_{4}=0.2$, and $\tau_{5}=0.1$. It is clear that bipartite consensus can be achieved when the input delays are below the upper bound of the allowable delay (see Figure 2). Let $\tau_{1}=0.4, \tau_{2}=0.3, \tau_{3}=0.4, \tau_{4}=0.3$, and $\tau_{5}=0.6$. The bipartite consensus cannot be achieved when one of input delays exceeds the upper bound of the allowable delay (see Figure 3).

Example 2. We will test and verify the results obtained by a multiagent system with six agents in this section. The initial conditions are randomly set and the topology is shown in Figure 4. The maximum eigenvalue of $L_{s}$ is $\lambda_{\max }=7.9101+1.3498 i$. Let $k_{1}=1$ and $k_{2}=1$.According to Theorem 2, we obtain $\tau^{*}<0.2$. When $\tau_{1}=0.17, \tau_{2}=0.16$, $\tau_{3}=0.15, \tau_{4}=0.14, \tau_{5}=0.13$, and $\tau_{6}=0.12$, bipartite consensus can be achieved (see Figure 5). When $\tau_{1}=0.22, \tau_{2}=$ $0.14, \tau_{3}=0.15, \tau_{4}=0.16, \tau_{5}=0.15$, and $\tau_{6}=0.14$, bipartite consensus cannot be achieved (see Figure 6).

\section{Conclusions}

Different from the previous work, we consider bipartite consensus of heterogeneous multiagent systems with diverse input delays. Based on the matrix theory and the frequency domain theory, the maximum input delay for the systems to achieve bipartite consensus is obtained. The future work will extend the existing work to time-varying input delays.

\section{Data Availability}

No data were used to support this study.

\section{Conflicts of Interest}

The authors declare that there are no conflicts of interest regarding the publication of this paper.

\section{Acknowledgments}

This work was supported by the Guangxi Key Laboratory of Automatic Detecting Technology and Instruments (YQ20208), National Natural Science Foundation of China (61963006 and 61563006), Natural Science Foundation of Guangxi Province (2018GXNSFAA050029 and 2018GXNSFAA294085), Guangxi Key Laboratory of Automobile Components and Vehicle Technology (2017GKLACVTZZ02), Innovation Project of Guangxi University of Science and Technology Graduate Education (GKYC201906), and Guangxi One Thousand Young and Middle-Aged College and University Backbone Teachers Cultivation Program. 


\section{References}

[1] R. Olfati-Saber and R. M. Murray, "Consensus problems in networks of agents with switching topology and time-delays," IEEE Transactions on Automatic Control, vol. 49, no. 9, pp. 1520-1533, 2004.

[2] X. Dong, B. Yu, Z. Shi, and Y. Zhong, "Time-varying formation control for unmanned aerial vehicles: theories and applications," IEEE Transactions on Control Systems Technology, vol. 23, no. 1, pp. 340-348, 2015.

[3] S.-L. Du, X.-M. Sun, M. Cao, and W. Wang, "Pursuing an evader through cooperative relaying in multi-agent surveillance networks," Automatica, vol. 83, pp. 155-161, 2017.

[4] C. Song, L. Liu, G. Feng, and S. Xu, "Coverage control for heterogeneous mobile sensor networks on a circle," Automatica, vol. 63, pp. 349-358, 2016.

[5] Z.-X. Li and H.-B. Ji, "Robust delay-dependent Ho consensus control for multi-agent systems with input delays," Acta Automatica Sinica, vol. 40, no. 11, pp. 2556-2562, 2014.

[6] T. Qi, L. Qiu, and J. Chen, "MAS consensus and delay limits under delayed output feedback," IEEE Transactions on Automatic Control, vol. 62, no. 9, pp. 4660-4666, 2017.

[7] X. Xu, L. Liu, and G. Feng, "Consensus of discrete-time linear multiagent systems with communication, input and output delays," IEEE Transactions on Automatic Control, vol. 63, no. 2, pp. 492-497, 2018.

[8] Y.-H. Lan, B. Wu, Y.-X. Shi, and Y.-P. Luo, "Iterative learning based consensus control for distributed parameter multiagent systems with time-delay," Neurocomputing, vol. 357, pp. 77-85, 2019.

[9] J. Liu, Y. Zhang, C. Sun, and Y. Yu, "Fixed-time consensus of multi-agent systems with input delay and uncertain disturbances via event-triggered control," Information Sciences, vol. 480, pp. 261-272, 2019.

[10] F. Ye, W. Zhang, and L. Ou, "H2 consensus control of timedelayed multi-agent systems: a frequency-domain method," ISA Transactions, vol. 66, pp. 437-447, 2017.

[11] F. Ye, B. Sun, L. Ou, and W. Zhang, "Disturbance observerbased control for consensus tracking of multi-agent systems with input delays from a frequency domain perspective," Systems \& Control Letters, vol. 114, pp. 66-75, 2018.

[12] A. S. Morteza, "Networks, crowds and markets: reasoning about a highly connected world," Journal of the Royal Statistical Society, vol. 175, no. 4, p. 1073, 2012.

[13] C. Parisien, C. H. Anderson, and C. Eliasmith, "Solving the problem of negative synaptic weights in cortical models," Neural Computation, vol. 20, no. 6, pp. 1473-1494, 2008.

[14] C. Altafini, "Consensus problems on networks with antagonistic interactions," IEEE Transactions on Automatic Control, vol. 58, no. 4, pp. 935-946, 2013.

[15] J. P. Hu, Z. H. Xiao, Y. L. Zhou, and J. Y. Yu, "Formation control over antagonistic networks," in Proceedings of the 32nd Chinese Control Conference, pp. 6879-6884, Xi'an, China, 2013.

[16] J. Qin, W. Fu, W. X. Zheng, and H. Gao, "On the bipartite consensus for generic linear multiagent systems with input saturation," IEEE Transactions on Cybernetics, vol. 47, no. 8, pp. 1948-1958, 2017.

[17] X. Gong, J. J. R. Liu, Y. M. Wang, and Y. K. Cui, "Distributed finite-time bipartite consensus of multi-agent systems on directed graphs: theory and experiment in nano-quadcopters formation," Journal of the Franklin Institute, In press, 2020.

[18] P. L. Li, Y. Liu, Y. D. Zhao, and J. Yang, "Consensus control for second-order multi-agent with time-delay on antagonistic networks," in Proceedings of the 35th Chinese Control Conference, pp. 8066-8071, Chengdu, China, 2016.

[19] H. Tian, C. L. Liu, and G. Y. Liu, "Bipartite consensus problem of second-order Multi-agent systems with disturbances," in 15th International Conference on Control, Automation, Robotics and Vision, Singapore, Singapore, November 2018.

[20] X. Guo, J. Lu, A. Alsaedi, and F. E. Alsaadi, "Bipartite consensus for multi-agent systems with antagonistic interactions and communication delays," Physica A: Statistical Mechanics and Its Applications, vol. 495, pp. 488-497, 2018.

[21] H. Wu, K. Gao, and B. R. An, "A class of second-order consensus protocol in multi-agent systems with multiple input delays," Journal of Systems Science and Complexity, vol. 32, no. 5, pp. 1-10, 2019.

[22] J. Ling, J. Liang, and M. Du, "Bipartite consensus problems on Second-order signed networks with heterogeneous topologies," IEEE Access, vol. 8, pp. 39420-39427, 2020.

[23] B. Sourav and P. Suraijt, "Lag-bipartite consensus of linear multi-agent systems under saturating input over signed graph," IET Control Theory \& Applications, vol. 13, no. 3, pp. 434-443, 2019.

[24] J. Liu, H. Li, and J. Luo, "Bipartite consensus in Networked Euler-Lagrange systems with uncertain parameters under a Cooperation-Competition network topology," IEEE Control Systems Letters, vol. 3, no. 3, pp. 494-498, 2019.

[25] H. Zhang, Y. Cai, Y. Wang, and H. Su, "Adaptive bipartite event-triggered output consensus of heterogeneous linear multiagent systems under fixed and switching topologies," IEEE Transactions on Neural Networks and Learning Systems, pp. 1-15, 2020.

[26] Y. L. Cai, H. G. Zhang, Y. Liu, and Q. He, "Distributed bipartite finite-time event-triggered output consensus for heterogeneous linear multi-agent systems under directed signed communication topology," Applied Mathematics and Computation, vol. 378, pp. 1-18, 2020.

[27] E. Li, Q. Ma, and G. Zhou, "Bipartite output consensus for heterogeneous linear multi-agent systems with fully distributed protocol," Journal of the Franklin Institute, vol. 356, no. 5, pp. 2870-2884, 2019.

[28] Q. Ma, G. Zhou, and E. Li, “Adaptive bipartite output consensus of heterogeneous linear multi-agent systems with antagonistic interactions," Neurocomputing, vol. 373, pp. 50-55, 2020.

[29] J. Yu and L. Wang, "Group consensus of multi-agent systems with directed information exchange," International Journal of Systems Science, vol. 43, no. 2, pp. 334-348, 2012.

[30] G. G. Wen, Y. G. Yu, Z. X. Peng, and W. Hu, "Dynamical group consensus of heterogeneous multi-agent systems with input time delays," Neurocomputing, vol. 175, pp. 278-286, 2015.

[31] C. L. Liu and F. Liu, "Dynamical consensus seeking of heterogeneous multi-agent systems under input delays," International Journal of Communication Systems, vol. 26, pp. 1243-1258, 2012.

[32] L. Lin, B. Francis, and M. Maggiore, "Necessary and sufficient graphical conditions for formation control of unicycles," IEEE Transactions on Automatic Control, vol. 50, no. 1, pp. 121-127, 2005.

[33] W. Ren and E. Atkins, "Distributed multi-vehicle coordinated control via local information exchange," International Journal of Robust \& Nonlinear Control, vol. 17, no. 10-11, pp. 10021033, 2007.

[34] L. H. Ji, X. H. Yu, and C. J. Li, "Group consensus for heterogeneous multi-agent systems in the competition networks 
with input time delay," IEEE Transactions on Systems, Man, and Cybernetics: Systems, pp. 1-9, 2018.

[35] H. Ye, M.-M. Li, W.-G. Luo, and Y.-X. Qin, "Finite-Time consensus of heterogeneous multi-agent systems without velocity measurements and with disturbances via integral sliding mode control," IEEE Access, vol. 6, pp. 62255-62260, 2018.

[36] Y. J. Hong, J. P. Hu, and L. Gao, "Tracking control for multiagent consensus with an active leader and variable topology," Automatica, vol. 42, no. 7, pp. 1177-1182, 2006. 